\title{
Associations between FAS rs2234767 and FASL rs763III0 polymorphisms and the risk of lung cancer: a meta-analysis of 39,736 subjects
}

This article was published in the following Dove Press journal:

OncoTargets and Therapy

6 April 2016

Number of times this article has been viewed

\author{
Xiongjie $\mathrm{Yu}^{1, *}$ \\ Yanli $\mathrm{Li}^{2}$,* \\ Yuandong Yu' \\ Jinhua Lei' \\ Guoxing Wan' \\ Fengjun $\mathrm{CaO}^{\prime}$ \\ 'Department of Oncology, \\ ${ }^{2}$ Department of Endocrinology, \\ Renmin Hospital, Hubei University \\ of Medicine, Shiyan, Hubei, People's \\ Republic of China \\ *These authors contributed equally \\ to this work
}

Background: Previous studies have investigated the associations between the common polymorphisms in FAS/FASL genes and lung cancer risk; however, the results remain inconsistent and inconclusive. Hence, we performed a meta-analysis to reassess the relationships between FAS rs2234767 and FASL rs763110 polymorphisms and the risk of lung cancer.

Methods: Eligible studies retrieved by an electronic search were pooled to calculate the strength of the associations using the odds ratio (OR) and 95\% confidence interval (95\% CI).

Results: A total of 13 case-control studies involving 39,736 subjects (9,237 cases and 10,838 controls on FAS rs2234767 and 8,957 cases and 10,704 controls on FASL rs763110) were included in the meta-analysis. The results showed a significant association between FAS rs2234767 polymorphism and increased risk of lung cancer (A vs G: OR $=1.07,95 \%$ $\mathrm{CI}=1.01-1.13$; AA vs GG: OR $=1.23,95 \% \mathrm{CI}=1.06-1.43$; AA vs GA + GG: OR $=1.24,95 \%$ $\mathrm{CI}=1.08-1.43$ ). Similar association was also observed in Asian population (AA vs GA + GG: $\mathrm{OR}=1.30,95 \% \mathrm{CI}=1.01-1.67$ ) and in the studies with large sample size (A vs $\mathrm{G}: \mathrm{OR}=1.07$, 95\% CI $=1.00-1.14$; AA vs GG: OR $=1.30,95 \%$ CI $=1.07-1.58$ ). However, no significant association between FASL rs763110 polymorphism and lung cancer risk was found other than in the Asian population (CC vs TC + TT: $\mathrm{OR}=1.35,95 \% \mathrm{CI}=1.01-1.80$ ).

Conclusion: The meta-analysis indicated that $F A S$ rs 2234767 polymorphism was significantly associated with an increased risk of lung cancer and FASL rs763110 polymorphism may not contribute to susceptibility to lung cancer other than in Asian population.

Keywords: FAS, FAS ligand, polymorphism, lung cancer, meta-analysis, risk, apoptosis

\section{Introduction}

Apoptosis is a genetically mediated physiological mechanism of programmed cell death, playing a vital role in the regulation of cell growth, homeostasis, and development of adult multicellular organisms, and elimination of unwanted or potentially dangerous cells. ${ }^{1,2}$ However, defects in the regulation of apoptosis result in unchecked cell growth and proliferation, causally contributing to tumorigenesis. ${ }^{3}$ Acquired ability to resist apoptosis is one of the hallmarks of malignancies including lung cancer, which usually arises from the genetic alterations of apoptotic genes such as FAS, FASL, TNF, TP53, Bcl-2, and so on. ${ }^{4-6}$

Acting as an important member of the tumor necrosis factor superfamily, FAS (also known as TNFSF6, CD95, or APO-1, located on chromosome 10q24.1), a cell surface receptor, has been delineated as a primary contributor to programmed cell death by the interaction with its natural ligand FASL (also CD95L, located on chromosome 1q23) to initiate the extrinsic pathway of apoptosis. ${ }^{7,8}$ In the FAS/FASL signaling 
system, FAS receptor is found to be extensively expressed in diverse tissues, while expression of FASL is restricted on the cells within the immune system, such as activated T-cells and natural killer cells. ${ }^{9}$ Decreased expression of FAS and/ or elevated expression of FASL are frequently observed in a variety of cancers including lung cancer in numerous studies, suggesting the potential importance of FAS/FASL signaling system in the formation of malignancy. ${ }^{10-12}$ As was strongly evidenced, decreased expression of FAS may protect tumor cells from apoptosis induced by antitumor immune responses, but increased expression of FASL may facilitate the ability of tumor cells to counterattack the immune system by damaging the FAS-sensitive lymphocytes and escape immunologic surveillance, thereby contributing to the development of cancer. $^{13-16}$

Recently, two common polymorphisms (a G to A transition at position -1,377 in $F A S$ gene, rs2234767 and a $\mathrm{T}$ to $\mathrm{C}$ transition at position -844 in FASL gene, rs763110) in the promoter regions of FAS and FASL have been found to be linked to the changeable expressions of the two genes and often associated with the altered risk of lung cancer. ${ }^{9,17}$ However, the results reported in previous studies regarding associations between the two common polymorphisms and the risk of lung cancer remain inconsistent and inconclusive, which may be partially attributed to the limited sample size of some published studies and variation in ethnicities. Accordingly, to derive a more precise estimation, we performed a meta-analysis with all available studies to comprehensively reevaluate the concerned associations.

\section{Materials and methods}

\section{Literature search}

A comprehensive electronic search in an attempt to retrieve relevant studies regarding the associations of $F A S$ rs 2234767 and FASL rs763110 polymorphisms with the risk of lung cancer was carried out in PubMed, Embase, Web of Science, Google Scholar, Cochrane Library, China National Knowledge Infrastructure, and Chinese Biomedicine Database (up to December 2015). We developed the search strategy using the following keywords ("FAS/CD95/TNFSF6/APO-1", "FASL/CD95L", "polymorphism/polymorphisms/variant/ genotype", and "lung cancer/lung carcinoma") in various combinations with the limits humans and articles in English or Chinese. Reference lists of the identified articles were also further checked for additional potentially eligible studies. In case of duplication, only the study with the largest sample size was selected. For the studies conducted in different ethnicities, each study should be treated independently.

\section{Inclusion criteria and data extraction}

The eligible studies for inclusion must satisfy the following criteria: 1) case-control studies; 2) studies evaluating the associations of FAS rs2234767 and FASL rs763110 polymorphisms with the risk of lung cancer; 3 ) diagnoses of all cases pathologically confirmed in the studies; 4) studies presenting sufficient information to calculate the odds ratios (ORs) with their 95\% confidence intervals (CIs); and 5) studies with available extracted frequencies in case and control. Studies without control or with incomplete data, familybased studies, or case reports, editorials, review articles, and meta-analysis were excluded. Data were independently extracted by two investigators using a standardized form. Any discrepancy between the two investigators was settled by discussion and consultation with a third investigator until a consensus was reached. The following information was extracted from each study: first author, year of publication, country, ethnicity, sample size, source of control, genotype method, allele or genotype frequencies in cases and controls, and evidence of Hardy-Weinberg equilibrium.

\section{Statistical analysis}

Individual ORs with 95\% CIs were utilized to evaluate the strength of associations between $F A S$ rs 2234767 and FASL rs763110 polymorphisms and lung cancer risk using Review Manager version 5.2 software (Cochrane Collaboration, Oxford, UK; http://www.cochrane.org/ software/revman.htm) in five genetic models (allele model, homozygote model, heterozygote model, dominant model, and recessive model). The significance of pooled OR was determined by Cochrane $Z$ test $(P<0.05$ was considered significant) using a fixed-effect model or random-effect model according to the heterogeneity across studies. ${ }^{18}$ The heterogeneity between studies was measured by the $Q$-test and $I^{2}$ statistics, and the random-effect model was applied if the heterogeneity was significant $\left(P_{\mathrm{h}}<0.1\right)$; otherwise, the fixed-effect model was used. ${ }^{19-21}$ In addition, the subgroup analysis was performed based on the ethnicity and sample size. The study with more than 2,000 subjects enrolled was regarded as having a large sample size; if not, it was defined as having small sample size. Moreover, sensitivity analysis by sequential omission of each individual study was performed to assess the stability of results. Potential publication bias was evaluated graphically by Begg's funnel plots, which was further assessed by Egger's linear regression test using Stata 12.0 software (StataCorp LP, College Station, TX, USA) and $P<0.05$ was considered statistically significant. ${ }^{22,23}$ 


\section{Results}

\section{Characteristics of included studies}

Based on the search strategy, 39 potentially relevant articles were retrieved by the initial research. However, 13 individual studies from six publications with a total of 39,736 subjects were finally enrolled in the meta-analysis, according to the selection criteria. ${ }^{1,3,6,9,24,25}$ The flow chart of study selection is shown in Figure 1. The study by Truong et $\mathrm{al}^{25}$ investigated the associations of FAS rs2234767 and FASL rs 763110 polymorphisms with the risk of lung cancer in Asian and Caucasian populations, respectively, which were treated independently. Therefore, there were seven case-control studies involving 9,237 cases and 10,838 controls focusing on the association between $F A S$ rs2234767 polymorphism and susceptibility to lung cancer, among which four and three studies were conducted in Asian and Caucasian populations, respectively. Also, six case-control studies involving 8,957 cases and 10,704 controls were included to reevaluate the association between FASL rs763110 polymorphism and the risk of lung cancer, of which there were four and two studies for Asian and Caucasian populations, respectively. No significant deviation from Hardy-Weinberg equilibrium was reported in the controls of all studies, except for the studies by Gormus et al, ${ }^{24}$ Zhang et al, ${ }^{9}$ and
Truong et al. ${ }^{25}$ The main characteristics of the included studies are listed in Table 1.

\section{Meta-analysis results}

The main results of the meta-analysis are shown in Table 2 and Figure 2. Overall, a significant association between FAS rs2234767 polymorphism and increased risk of lung cancer was observed in allele model (A vs G: OR $=1.07,95 \% \mathrm{CI}=1.01-1.13$ ), homozygote model (AA vs GG: $\mathrm{OR}=1.23,95 \% \mathrm{CI}=1.06-1.43)$, and recessive model (AA vs GA + GG: OR $=1.24,95 \% \mathrm{CI}=1.08-1.43$ ). In the subgroup analysis based on ethnicity, the FAS rs2234767 polymorphism was associated with an increased risk of lung cancer (AA vs GA + GG: $\mathrm{OR}=1.30,95 \% \mathrm{CI}=1.01-1.67$ ) in Asian population, but not in Caucasian population. In addition, the subgroup analysis by sample size represented an increased lung cancer risk associated with FAS rs2234767 polymorphism (A vs $\mathrm{G}$ : OR $=1.07,95 \% \mathrm{CI}=1.00-1.14$; AA vs $\mathrm{GG}: \mathrm{OR}=1.30,95 \% \mathrm{CI}=1.07-1.58$ ) in large sample size rather than small sample size.

For FASL rs763110 polymorphism, no significant overall risk of lung cancer was revealed in any genetic model. However, an increased risk of lung cancer associated with this variant was detected in Asian population in recessive

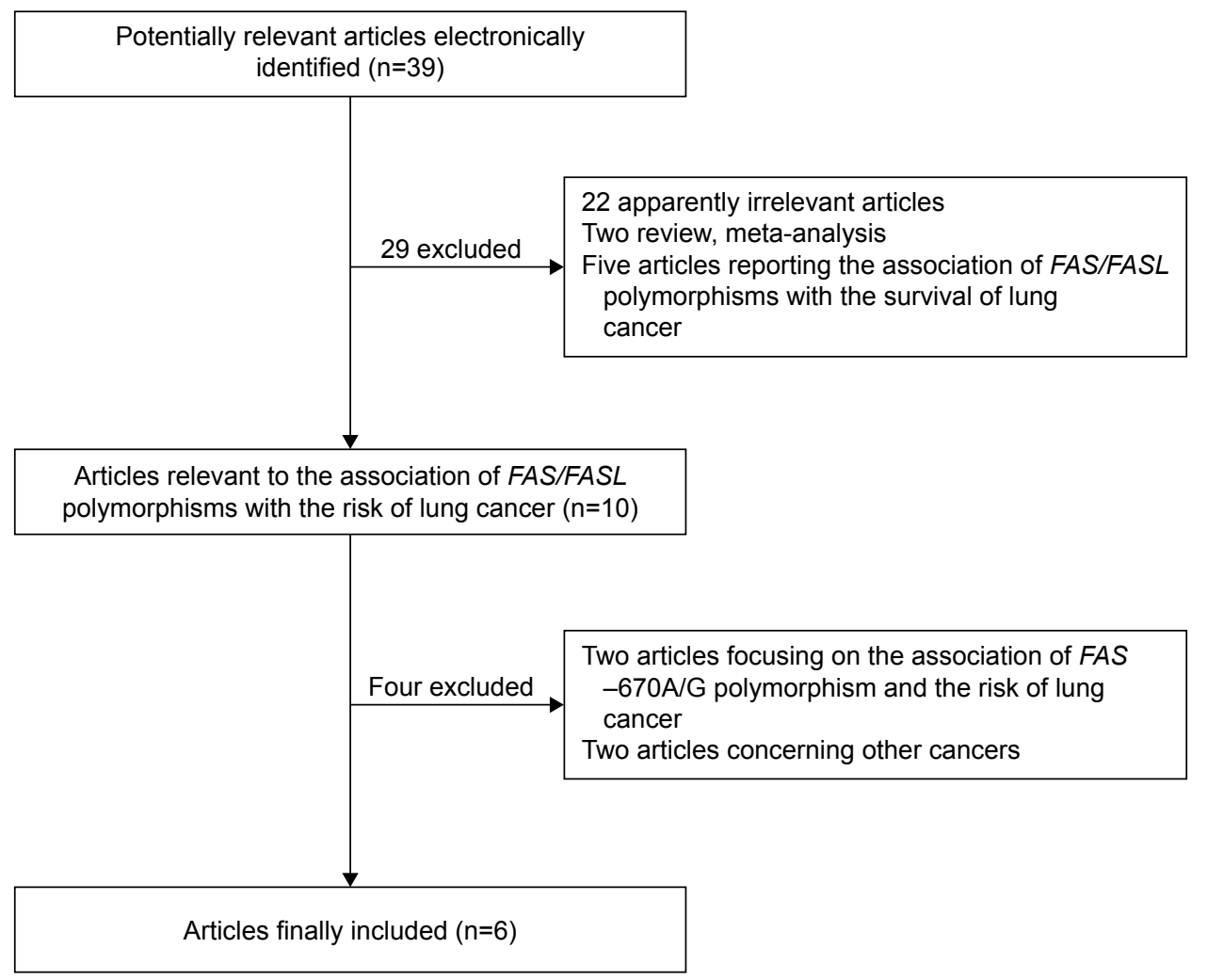

Figure I Flowchart of the selection procedure. 

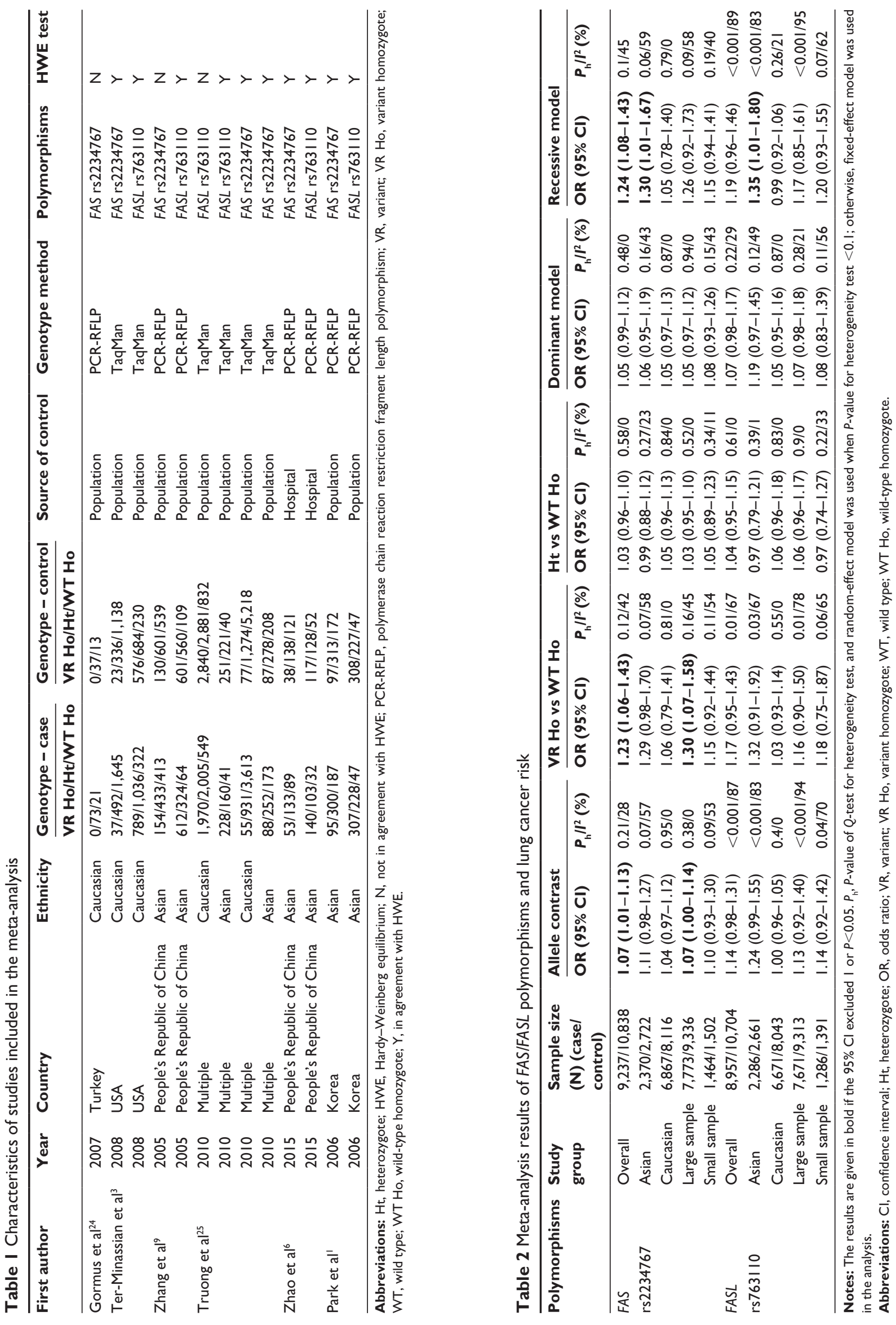


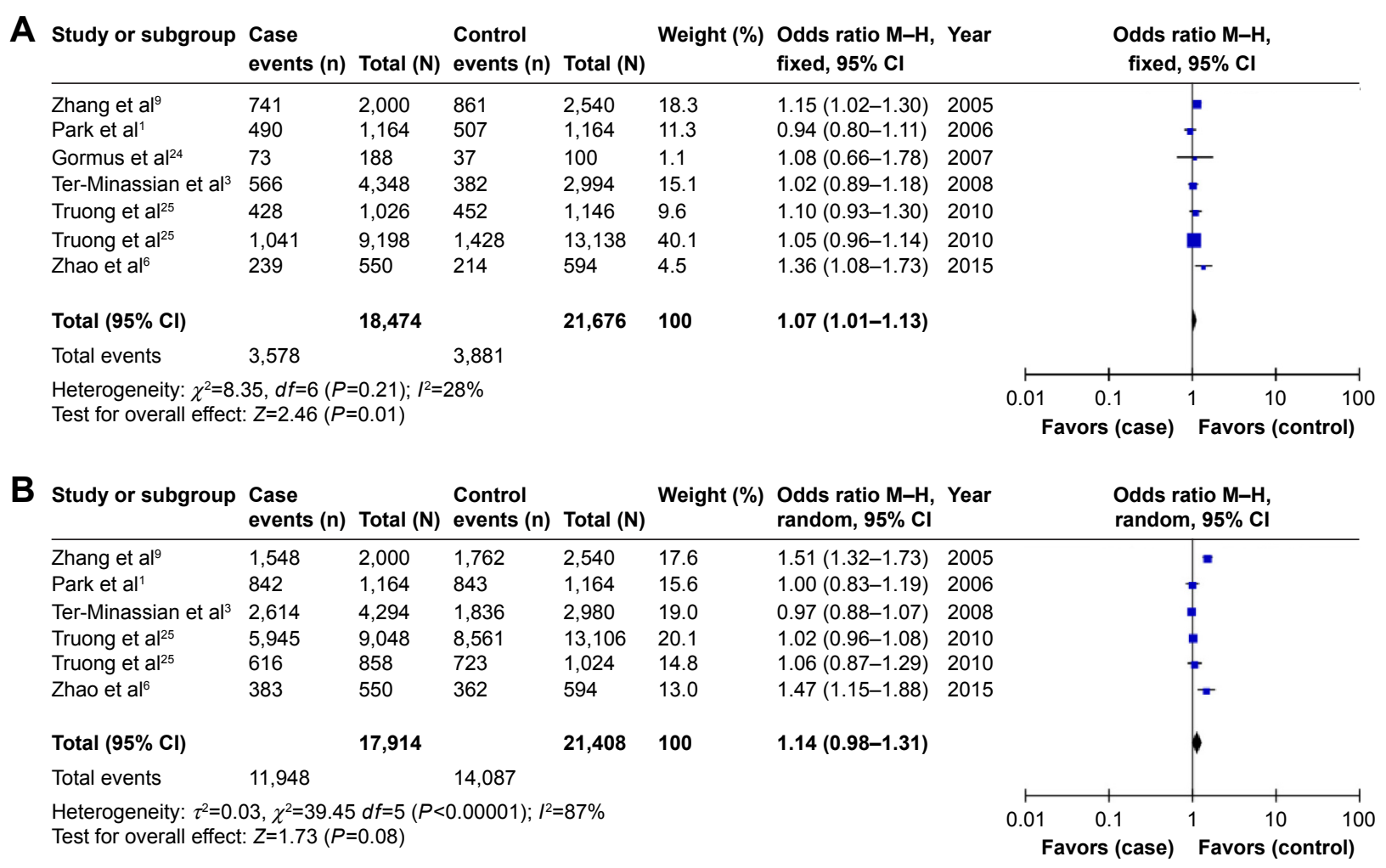

Figure 2 Forest plots of lung cancer risk associated with variants of FASIFASL gene in the allele contrast. Notes: (A) FAS -1377G/A, rs2234767; (B) FASL rs763। I0, rs763। I0.

Abbreviations: $\mathrm{Cl}$, confidence interval; $d f$, degrees of freedom; $\mathrm{M}-\mathrm{H}$, Mantel-Haenszel.

model $(\mathrm{CC}$ vs TC + TT: OR $=1.35,95 \% \mathrm{CI}=1.01-1.80)$ in the subgroup analysis based on ethnicity, while such an association was not found in Caucasian population. Similarly, no obvious association between FASL rs763110 polymorphism and susceptibility to lung cancer was indicated in the stratification analysis by sample size.

\section{Sensitivity analysis and publication bias test}

Sensitivity analysis by sequential omission of each individual study was performed to assess the stability of the results. In the present study, no quantitative alternation of overall ORs were observed under any genetic model in the sensitivity analysis for the association between $F A S$ rs 2234767 polymorphism and the risk of lung cancer, with the exception of the allele contrast model by excluding the study by Zhang et al ${ }^{9}$ (pooled $\mathrm{OR}=1.05,95 \% \mathrm{CI}=0.99-1.12, P=0.1)$, the homozygote by excluding the studies by Zhang et $\mathrm{al}^{9}$ (pooled OR $=1.12$, 95\% CI $=0.94-1.33, P=0.22$ ), Ter-Minassian et al $^{3}$ (pooled $\mathrm{OR}=1.23,95 \% \mathrm{CI}=0.98-1.55, P=0.08$ ), and Truong et $\mathrm{al}^{25}$ (pooled $\mathrm{OR}=1.22,95 \% \mathrm{CI}=0.95-1.56, P=0.12$ ), as well as the recessive model (pooled OR $=1.12,95 \% \mathrm{CI}=0.95-1.32$, $P=0.19$ for Zhang et al, ${ }^{9}$ pooled $\mathrm{OR}=1.24,95 \% \mathrm{CI}=1.00$ 1.54, $P=0.06$ for Ter-Minassian et al, ${ }^{3}$ pooled $\mathrm{OR}=1.24$,
$95 \% \mathrm{CI}=0.98-1.57, P=0.08$ for Truong et $\left.\mathrm{al}^{25}\right)$. Nevertheless, most results of the sensitivity analysis suggested at least a borderline significance of this association. For the FASL rs 763110 polymorphism, the significance of the pooled OR was not affected excessively in any of the genetic models, indicating the robustness of the results. Furthermore, as shown in Figure 3, no obvious asymmetry was observed in Begg's funnel plots for the two studied polymorphisms and no supportive evidence was provided for potential publication bias by the Egger's test (dominant model: $P=0.513$ for $F A S$ rs 2234767 polymorphism and $P=0.592$ for $F A S L$ rs763110 polymorphism), further supporting the absence of evident publication bias.

\section{Discussion}

The present study involving 39,736 subjects was the first to comprehensively evaluate the associations between two common polymorphisms in FAS/FASL signal and susceptibility to lung cancer, demonstrating an increased risk of lung cancer associated with $F A S$ rs 2234767 polymorphism, whereas it suggested no significant association between FASL rs763110 polymorphism and lung cancer risk other than in an Asian population.

Over decades, accumulative evidence has shed light on the advances in basic lung cancer research that apoptosis and 

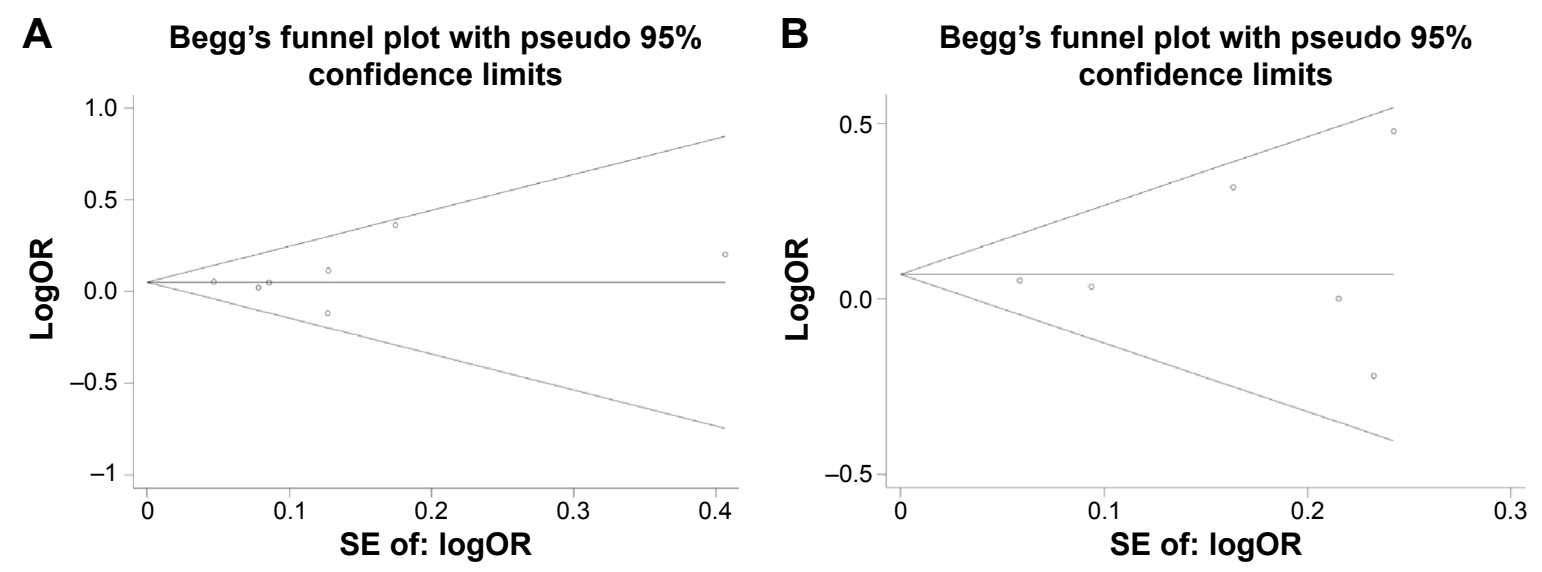

Figure 3 Begg's funnel plots for publication bias test on the associations of FAS/FASL gene polymorphisms with lung cancer risk in the dominant contrast. Notes: (A) FAS - 1377G/A, rs2234767; (B) FASL rs763।10, rs763।10.

Abbreviations: OR, odds ratio; $\mathrm{SE}$, standard error.

apoptosis-related genes may contribute vastly to the development of the malignant phenotype by conferring enhanced ability to resist apoptotic stimuli to some tumor cells or by inducing apoptosis of tumor-specific lymphocytes. ${ }^{26,27}$ Remarkably, the discovery of FAS/FASL signaling system primarily involved in apoptosis has enabled us to recognize carcinogenesis of apoptosis-related genes, with aberrant expressions of FAS and FASL observed in lung cancer, which was supported by numerous lines of evidence. ${ }^{11,28}$ In this regard, the existence of two paths for the FAS/FASL system in lung cancer has been widely accepted: FASL on T-lymphocytes is able to promote apoptosis in FASexpressing lung cancer cells through cell-mediated cytotoxic reactions against cancer; in contrast, lung cancer cells can escape from the immune system by downregulating FAS expression and/or inducing the apoptosis of lymphocytes by the expression of FASL. ${ }^{29-31}$ Given the important role of FAS/FASL system in lung cancer, it is biologically plausible to propose that several polymorphisms in $F A S / F A S L$ genes may be significant in the genetic susceptibility to lung cancer on account of the fact that these polymorphisms possess the potential to alter the expression of FAS and/or FASL. ${ }^{6}$ For instance, the $F A S$ rs 2234767 polymorphism was found to be capable of disrupting Sp1 transcription factor binding sites, thus diminishing the promoter activity and decreasing the expression of FAS gene; ${ }^{32}$ the $\mathrm{C}$ allele of the FASL$844 \mathrm{~T}>\mathrm{C}$ polymorphism could create a binding site for a transcription factor, CAAT/enhancer-binding protein, and thus is significantly related to increased basal expression of FASL, ${ }^{33}$ which are both able to result in altered risk of lung cancer via the aforementioned mechanism. Nevertheless, current studies investigating the relationships between FAS rs2234767 and FASL rs763110 polymorphisms and the risk of lung cancer reported inconsistent results, which prompts us to perform a meta-analysis to reassess the associations with a powerful approach.

In this meta-analysis, 13 published studies with a total of 39,736 subjects were enrolled to determine the associations between the two potentially functional polymorphisms within FAS and FASL and the lung cancer risk. Overall, the study suggested a significant association between FAS rs2234767 polymorphism and increased risk of lung cancer, particularly evident in Asian population, which was in keeping with the findings by $\mathrm{Xu}$ et al. ${ }^{34}$ They performed a meta-analysis to investigate the association of $F A S$ rs 2234767 polymorphism with overall cancer risk and reported that the polymorphism was associated with a higher cancer risk including lung cancer and the overall cancer risk associated with the polymorphism was particularly evident in Asian population, although a smaller sample size was included in their study compared to that in the current study. Overall, our study along with the previous study has further validated this association in a more confident manner. Also, the subgroup analysis based on sample size in the present study revealed that this association was particularly evident in a lager sample size, suggesting the importance of sample size in determining the concerned association. Furthermore, the association of FASL rs 763110 polymorphisms with the risk of lung cancer was also assessed in the present study; however, we failed to show a significant association between this polymorphism and the overall risk of lung cancer. But an elevated lung cancer risk associated with FASL rs763110 variant was observed, although modest, in Asian population but not in Caucasian population, indicating the possible contribution of ethnicity variation to the association between $F A S L$ rs 763110 polymorphisms with lung cancer risk. However, this association should be further 
verified with high-quality and homogeneous studies in light of the obvious heterogeneity in several genetic models.

In spite of the powerful approach, several limitations of the study remain to be taken into consideration. First, the included studies were all reported in English and Chinese; some other studies in other languages satisfying the inclusion criteria would have been inevitably omitted. Second, our results are based on unadjusted estimates; some important compounding factors such as smoking, age, and occupation, which may significantly impact the risk of lung cancer, were not considered due to lack of original data. Third, the significance of the studied polymorphisms in different subtypes of lung cancer may be inconsistent; however, we are unable to perform subgroup analysis by subtype due to the limited information because the subtypes of lung cancer in most studies were not clearly clarified.

\section{Conclusion}

In summary, the meta-analysis further indicated that $F A S$ rs2234767 polymorphism was significantly associated with an increased risk of lung cancer and FASL rs763110 polymorphism may not contribute to susceptibility to lung cancer other than in Asian population; however, it remains to be further verified considering the modesties of the association and the heterogeneity presented. A definite conclusion with more confidence should be drawn with more high-quality and -uniformity case-control studies in different ethnicities in the future. Nevertheless, these findings may raise the public awareness on the role of concerned polymorphisms in the development of lung cancer, the detection of which would certainly be one of the most promising strategies in preventing cancer by identifying the possible susceptible populations.

\section{Disclosure}

The authors report no conflicts of interest in this work.

\section{References}

1. Park SH, Choi JE, Kim EJ, et al. Polymorphisms in the FAS and FASL genes and risk of lung cancer in a Korean population. Lung Cancer. 2006; 54:303-308

2. Zhu J, Lu L, Cheng X, et al. Association between CD95L polymorphism and cervical cancer risk: evidence from a meta-analysis. Tumor Biol. 2014; 35:5137-5142.

3. Ter-Minassian M, Zhai R, Asomaning K, et al. Apoptosis gene polymorphisms, age, smoking and the risk of non-small cell lung cancer. Carcinogenesis. 2008;29:2147-2152.

4. Fan XQ, Guo YJ. Apoptosis in oncology. Cell Res. 2001;11:1-7.

5. Evan GI, Vousden KH. Proliferation, cell cycle and apoptosis in cancer. Nature. 2001;411:342-348.

6. Zhao H, Chen W, Du P, et al. FasL rs763110 and Fas -1377G/A: mutations of pulmonary adenocarcinoma in South China and their clinical significances. Tumor Biol. 2015;36:4319-4326.
7. Oehm A, Behrmann I, Falk W, et al. Purification and molecular cloning of the APO-1 cell surface antigen, a member of the tumor necrosis factor/nerve growth factor receptor superfamily. Sequence identity with the Fas antigen. J Biol Chem. 1992;267:10709-10715.

8. Suda T, Takahashi T, Golstein P, Nagata S. Molecular cloning and expression of the Fas ligand, a novel member of the tumor necrosis factor family. Cell. 1993;75:1169-1178.

9. Zhang X, Miao X, Sun T, et al. Functional polymorphisms in cell death pathway genes FAS and FASL contribute to risk of lung cancer. $J$ Med Genet. 2005;42:479-484.

10. Lee SH, Shin MS, Park WS, et al. Alterations of Fas (APO-1/CD95) gene in transitional cell carcinomas of urinary bladder. Cancer Res. 1999; 59:3068-3072.

11. Viard-Leveugle I, Veyrenc S, French LE, Brambilla C, Brambilla E. Frequent loss of Fas expression and function in human lung tumors with overexpression of FasL in small cell lung carcinoma. J Pathol. 2003; 201:268-277.

12. Gratas C, Tohma Y, Barnas C, Taniere P, Hainaut P, Ohgaki H. Up-regulation of Fas (APO-1/CD95) ligand and down-regulation of Fas expression in human esophageal cancer. Cancer Res. 1998;58: 2057-2062.

13. Gastman BR, Atarshi Y, Reichert TE, et al. Fas ligand is expressed on human squamous cell carcinomas of the head and neck, and it promotes apoptosis of T lymphocytes. Cancer Res. 1999;59: 5356-5364.

14. Koyama S, Koike N, Adachi S. Fas receptor counterattack against tumorinfiltrating lymphocytes in vivo as a mechanism of immune escape in gastric carcinoma. J Cancer Res Clin Oncol. 2001;127:20-26.

15. Bennett MW, O'Connell J, O’Sullivan GC, et al. The Fas counterattack in vivo: apoptotic depletion of tumor-infiltrating lymphocytes associated with Fas ligand expression by human esophageal carcinoma. J Immunol. 1998;160:5669-5675.

16. von Bernstorff W, Spanjaard RA, Chan AK, et al. Pancreatic cancer cells can evade immune surveillance via nonfunctional Fas (APO-1/ CD95) receptors and aberrant expression of functional Fas ligand. Surgery. 1999;125:73-84.

17. Li F, Liu Y, Fu T, Tong W, Zhang A. Associations of three common polymorphisms in CD95 and CD95L promoter regions with gastric cancer risk. Tumor Biol. 2013;34:2293-2298.

18. Higgins JP, Thompson SG. Quantifying heterogeneity in a metaanalysis. Stat Med. 2002;21:1539-1558.

19. Higgins JP, Thompson SG, Deeks JJ, Altman DG. Measuring inconsistency in meta-analyses. BMJ. 2003;327:557-560.

20. DerSimonian R, Laird N. Meta-analysis in clinical trials. Control Clin Trials. 1986;7:177-188.

21. Mantel N, Haenszel W. Statistical aspects of the analysis of data from retrospective studies of disease. J Natl Cancer Inst. 1959;22:719-748.

22. Begg CB, Mazumdar M. Operating characteristics of a rank correlation test for publication bias. Biometrics. 1994;50:1088-1101.

23. Egger M, Davey SG, Schneider M, Minder C. Bias in meta-analysis detected by a simple, graphical test. BMJ. 1997;315:629-634.

24. Gormus U, Ergen A, Yaylim-Eraltan I, et al. Fas-1377 A/G polymorphism in lung cancer. In Vivo. 2007;21:663-666.

25. Truong T, Sauter W, McKay JD, et al. International Lung Cancer Consortium: coordinated association study of 10 potential lung cancer susceptibility variants. Carcinogenesis. 2010;31:625-633.

26. Shimizu M, Kondo M, Ito Y, Kume H, Suzuki R, Yamaki K. Soluble Fas and Fas ligand provide new information on metastasis and response to chemotherapy in SCLC patients. Cancer Detect Prev. 2005;29: 175-180.

27. Liu K, Caldwell SA, Abrams SI. Immune selection and emergence of aggressive tumor variants as negative consequences of Fas-mediated cytotoxicity and altered IFN-gamma- regulated gene expression. Cancer Res. 2005;65:4376-4388.

28. Boldrini L, Faviana P, Pistolesi F, et al. Alterations of Fas (APO-1/CD 95) gene and its relationship with p53 in non small cell lung cancer. Oncogene. 2001;20:6632-6637. 
29. Griffith TS, Brunner T, Fletcher SM, Green DR, Ferguson TA. Fas ligand-induced apoptosis as a mechanism of immune privilege. Science. 1995;270:1189-1192.

30. Strand S, Hofmann WJ, Hug H, et al. Lymphocyte apoptosis induced by CD95 (APO-1/Fas) ligand-expressing tumor cells - a mechanism of immune evasion? Nat Med. 1996;2:1361-1366.

31. Reichmann E. The biological role of the Fas/FasL system during tumor formation and progression. Semin Cancer Biol. 2002;12:309-315.

32. Sun T, Miao X, Zhang X, Tan W, Xiong P, Lin D. Polymorphisms of death pathway genes FAS and FASL in esophageal squamous-cell carcinoma. J Natl Cancer Inst. 2004;96:1030-1036.
33. Wu J, Metz C, Xu X, et al. A novel polymorphic CAAT/enhancerbinding protein beta element in the FasL gene promoter alters Fas ligand expression: a candidate background gene in African American systemic lupus erythematosus patients. J Immunol. 2003;170:132-138.

34. Xu Y, He B, Li R, et al. Association of the polymorphisms in the Fas/FasL promoter regions with cancer susceptibility: a systematic review and meta-analysis of 52 studies. PLoS One. 2014;9:e90090.

\section{Publish your work in this journal}

OncoTargets and Therapy is an international, peer-reviewed, open access journal focusing on the pathological basis of all cancers, potential targets for therapy and treatment protocols employed to improve the management of cancer patients. The journal also focuses on the impact of management programs and new therapeutic agents and protocols on

\section{Dovepress}

patient perspectives such as quality of life, adherence and satisfaction. The manuscript management system is completely online and includes a very quick and fair peer-review system, which is all easy to use. Visit http://www.dovepress.com/testimonials.php to read real quotes from published authors. 